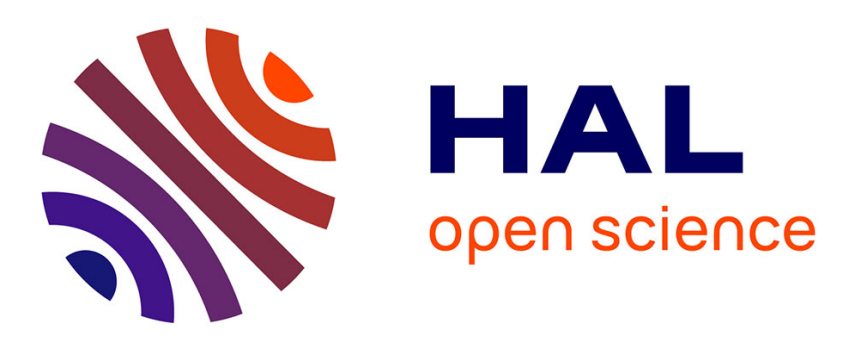

\title{
Visually Servoing a Gough-Stewart Parallel Robot Allows for Reduced and Linear Kinematic Calibration
}

Nicolas Andreff, Philippe Martinet

\section{To cite this version:}

Nicolas Andreff, Philippe Martinet. Visually Servoing a Gough-Stewart Parallel Robot Allows for Reduced and Linear Kinematic Calibration. ICINCO 2005 - 2nd International Conference on Informatics in Control, Automation and Robotics, Sep 2005, Barcelona, Spain. pp.119-124, 10.5220/0001174301190124. hal-02467466

\section{HAL Id: hal-02467466 https://hal.inria.fr/hal-02467466}

Submitted on 5 Feb 2020

HAL is a multi-disciplinary open access archive for the deposit and dissemination of scientific research documents, whether they are published or not. The documents may come from teaching and research institutions in France or abroad, or from public or private research centers.
L'archive ouverte pluridisciplinaire HAL, est destinée au dépôt et à la diffusion de documents scientifiques de niveau recherche, publiés ou non, émanant des établissements d'enseignement et de recherche français ou étrangers, des laboratoires publics ou privés. 


\title{
VISUALLY SERVOING A GOUGH-STEWART PARALLEL ROBOT ALLOWS FOR REDUCED AND LINEAR KINEMATIC CALIBRATION
}

\author{
Nicolas Andreff and Philippe Martinet \\ LAboratoire des Sciences et Matériaux pour l'Electronique et d'Automatique (LASMEA) \\ UMR 6602 CNRS-Université Blaise Pascal \\ 24 avenue des Landais, 63177 Aubire Cedex, France \\ Email: Nicolas.Andreff@ifma.fr, Philippe.Martinet@lasmea.univ-bpclermont.fr
}

\begin{abstract}
Keywords: parallel robot, visual servoing, redundant metrology, kinematic calibration.
Abstract: This paper focuses on the benefits of using computer vision to control a Gough-Stewart parallel robot. Namely, it is recalled that observing the legs of such a mechanism with a calibrated camera, thus following the redundant metrology paradigm, simplifies the control law. Then, we prove in this paper that such a control law depends on a reduced set of kinematic parameters (only those attached to the geometry of the robot base) and that these parameters can be obtained by solving a linear system. Moreover, we show that the camera can be calibrated without much experimental effort, simply using images of the robot itself. All this means that setting up the control system consists only in placing the camera in front of the robot.
\end{abstract}

\section{INTRODUCTION}

Parallel mechanism are such that there exist several kinematic chains (or legs) between their base and their end-effector. Therefore, they may exhibit a better repeatability (Merlet, 2000) than serial mechanisms but not a better accuracy (Wang and Masory, 1993), because of the large number of links and passive joints. There can be two ways to compensate for the low accuracy. The first way is to perform a kinematic calibration of the mechanism and the second one is to use a control law which is robust to calibration errors.

There exists a large amount of work on the control of parallel mechanisms ${ }^{1}$. In the focus of attention, Cartesian control is naturally achieved through the use of the inverse Jacobian which transforms Cartesian velocities into joint velocities. It is noticeable that the inverse Jacobian of parallel mechanisms does not only depend on the joint configuration (as for serial mechanisms) but also on the end-effector pose.

Consequently, one needs to be able to estimate or measure the latter. As far as we know, all the effort has been put on the estimation of the end-effector pose through the forward kinematic model and the joint measurements. However, this yields much trouble, related to the fact that in general, there is no

\footnotetext{
${ }^{1}$ See http://www-sop.inria.fr/coprin/equipe/merlet for a long list of references.
}

closed-form solution to the forward kinematic problem. Hence, one numerically inverts the inverse kinematic model, which is analytically defined for most of the parallel mechanisms. However, it is known (Merlet, 1990; Husty, 1996) that this numerical inversion requires high order polynomial root determination, with several possible solutions (up to 40 real solutions for a Gough-Stewart platform (Dietmaier, 1998)). Much of the work is thus devoted to solving this problem accurately and in real-time (see for instance (Zhao and Peng, 2000)), or to designing parallel mechanisms with analytical forward kinematic model (Kim and Tsai, 2002; Gogu, 2004). One of the promising paths lies in the use of the so-called metrological redundancy (Baron and Angeles, 1998), which simplifies the kinematic models by introducing additional sensors into the mechanism and thus yields easier control (Marquet, 2002).

Visual servoing (Espiau et al., 1992; Christensen and Corke, 2003) is known to robustify the Cartesian control of serial and mobile robots, with techniques ranging from position-based visual servoing (PBVS) (Martinet et al., 1996) (when the pose measurement is explicit) to image-based visual servoing (IBVS) (Espiau et al., 1992) (when it is made implicit by using only image measurements). Visual servoing techniques are very effective since they close the control loop on the vision sensor, which gives a direct view of the Cartesian space. This yields a high 
robustness to robot calibration errors. Indeed, these errors only appear in a Jacobian matrix but not in the regulated error.

Essentially, visual servoing techniques generate a Cartesian desired velocity which is converted into joint velocities by the robot inverse Jacobian. Hence, one can translate such techniques to parallel mechanisms as in (Koreichi et al., 1998; Kallio et al., 2000) by observation of the robot end-effector and the use of standard kinematic models. It is rather easier than in the serial case, since the inverse Jacobian of a parallel mechanism has a straightforward expression. Moreover, for parallel mechanisms, since the joint velocities are filtered through the inverse Jacobian, they are admissible, in the sense that they do not generate internal forces. More precisely, this is only true in the theoretical case. However, if the estimated inverse Jacobian used for control is close enough to the actual one, the joint velocities will be closely admissible, in the sense that they do not generate high internal forces. The only difficulty would come from the dependency of the inverse Jacobian to the Cartesian pose, which would need be estimated, but, as stated above, vision can also do that (DeMenthon and Davis, 1992; Lavest et al., 1998) ! Notice that this point pleads for PBVS rather than IBVS of parallel mechanisms.

Nevertheless, the direct application of visual servoing techniques assumes implicitly that the robot inverse Jacobian is given and that it is calibrated. Therefore, modeling, identification and control have small interaction with each other. Indeed, the model is usually defined for control using proprioceptive sensing only and does not foresee the use of vision for control, then identification and control are defined with the constraints imposed by the model. This is useful for modularity but this might not be efficient in terms of accuracy as well as of experimental set-up time.

On the opposite, instead of having identification and control being driven by the initial modeling stage, one could have a model taking into account the use of vision for control and hence for identification. To do so, a new way to use vision, which gathers the advantages of redundant metrology and of visual servoing and avoids most of their drawbacks was presented in (Andreff et al., 2005).

Indeed, adding redundant sensors is not always technically feasible (think of a spherical joint) and always requires either that the sensors are foreseen at design stage or that the mechanism is physically modified to install them after its building. Anyhow, there are then additional calibration parameters in the kinematic model and one needs to estimate them in order to convert redundant joint readings into a unit vector expressed in the appropriate reference frame. Moreover, observing the end-effector of a parallel mechanism by vision may be incompatible with its applica-

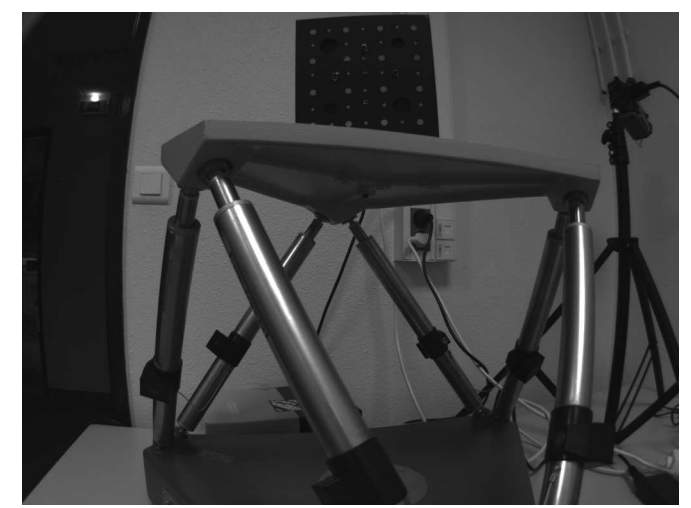

Figure 1: A Gough-Stewart platform observed by a camera with short focal length.

tion. For instance, it is not wise to imagine observing the end-effector of a machining tool. On the opposite, it should not be a problem to observe the legs of the mechanism, even in such extreme cases. Thereby one would turn vision from an exteroceptive sensor to a somewhat more proprioceptive sensor. This brings us back to the redundant metrology paradigm.

With such an approach, the control is made easier by measuring a major part of the robot inverse Jacobian, reducing the number of kinematic parameters to be identified. We will show in this paper that these remaining parameters can be estimated by solving a linear system.

The remainder of the paper is the following. Section 2 recalls the vision-based control of the GoughStewart robot. Section 3 discusses the actual choice of vision for such a control. Section 4 presents the calibration procedure for the whole system. Finally, simulation results and conclusion are given respectively in section 5 and section 6 .

\section{VISION-BASED KINEMATICS}

Consider the hexapod in Figure 1. It has 6 legs of varying length $q_{i}, i \in 1 . .6$, attached to the base by spherical joints located in points $\mathbf{A}_{i}$ and to the moving platform (end-effector) by spherical joints located in points $\mathbf{B}_{i}$. The inverse kinematic model of such an hexapod is given by

$$
\forall i \in 1 . .6, \quad q_{i}^{2}=\overrightarrow{\mathbf{A}_{i} \mathbf{B}_{i}^{T}} \overrightarrow{\mathbf{A}_{i} \mathbf{B}_{i}}
$$

expressing that $q_{i}$ is the length of vector $\overrightarrow{\mathbf{A}_{i} \mathbf{B}_{i}}$. This model can be expressed in any Euclidean reference frame. Hence, it can be expressed in the base frame $\mathcal{R}_{b}$, in the end-effector frame $\mathcal{R}_{e}$ or in the camera frame $\mathcal{R}_{c}$. Hereafter, the camera reference frame will be used and denoted by a left upper-script. 
According to (Andreff et al., 2005) and assuming a calibrated camera, one can express the vision-based kinematics of the hexapod expressed in the camera frame:

with

$$
\begin{aligned}
q_{i}{ }^{c} \underline{\mathbf{u}}_{i} & ={ }^{c} \mathbf{R}_{e}{ }^{e} \mathbf{B}_{i}+{ }^{c} \mathbf{t}_{e}-{ }^{c} \mathbf{B}_{i} \\
\dot{\mathbf{q}} & ={ }^{c} \mathbf{J}_{c}^{i n v}{ }^{c} \tau_{c} \\
{ }^{c} \mathbf{J}_{c}^{i n v} & =-\left[\begin{array}{cc}
{ }^{c} \underline{\mathbf{u}}_{1}^{T}\left({ }^{c} \mathbf{A}_{1} \times{ }^{c} \underline{\mathbf{u}}_{1}\right)^{T} \\
\vdots & \vdots \\
{ }^{c} \underline{\mathbf{u}}_{6}^{T} & \left({ }^{c} \mathbf{A}_{6} \times{ }^{c} \underline{\mathbf{u}}_{6}\right)^{T}
\end{array}\right]
\end{aligned}
$$

where ${ }^{c} \tau_{c}$ is the Cartesian velocity of the camera frame, considered as attached to the base frame and moving with respect to a fixed end-effector, expressed in itself and ${ }^{c} \underline{\mathbf{u}}_{i}, i=1 . .6$ are the unit vectors giving the pointing direction of each leg in the camera frame.

Then, one can derive a control law of the form:

$$
\begin{aligned}
\mathbf{e}_{i} & ={ }^{c} \underline{\mathbf{u}}_{i} \times{ }^{c} \underline{\mathbf{u}}_{d i} \\
E & =\left(\mathbf{e}_{1}^{T}, \ldots, \mathbf{e}_{6}^{T}\right)^{T} \\
\mathbf{P}\left({ }^{c} \underline{\mathbf{u}}_{i}\right) & =\left(\mathbf{I}_{3}-{ }^{c} \underline{\mathbf{u}}_{i}{ }^{c} \underline{\mathbf{u}}_{i}^{T}\right) \\
\mathbf{M}_{i}^{T} & =\mathbf{P}\left({ }^{c} \underline{\mathbf{u}}_{i}\right)\left[\mathbf{I}_{3}-\left[{ }^{c} \mathbf{A}_{i}+q_{i}{ }^{c} \underline{\mathbf{u}}_{i}\right]_{\times}\right] \\
\mathbf{N}_{i}^{T} & =\frac{1}{q_{i}}\left[\underline{\mathbf{u}}_{d i}\right] \times \mathbf{M}_{i}^{T} \\
\mathbf{N}^{T} & =\left(\mathbf{N}_{1}, \ldots, \mathbf{N}_{6}\right)^{T} \\
\dot{\mathbf{q}} & =-\widehat{\lambda}^{c} \widehat{\mathbf{J}_{c}^{i n v}} \widehat{\mathbf{N}}^{T} \operatorname{diag}(\mathbf{q}) E
\end{aligned}
$$

where the hat means that only an estimate can be used and []$_{\times}$denotes the antisymmetric matrix associated to the cross-product.

\section{DISCUSSION}

Here, the above control is discussed, with explicit regard to the sensing problem. Indeed, it does not absolutely imply that computer vision should be used for that.

\subsection{Why vision should be used rather than joint sensors}

There are three manners to measure or estimate the $\underline{\mathbf{u}}_{i}$ 's. The first one, which we discard of course immediately, is to estimate the end-effector pose with respect to the base by numerical inversion of the inverse kinematic model and then use (2) to obtain $\underline{\mathbf{u}}_{i}$.

The second manner is to place joint sensors in the $\mathbf{A}_{i}$ 's, so that they would deliver the pointing direction of the leg. This manner is valid since the $\underline{\mathbf{u}}_{i}$ 's would be measured and not estimated through a delicate numerical procedure as above. Nevertheless, it is, in our opinion, still not the correct manner. Indeed,

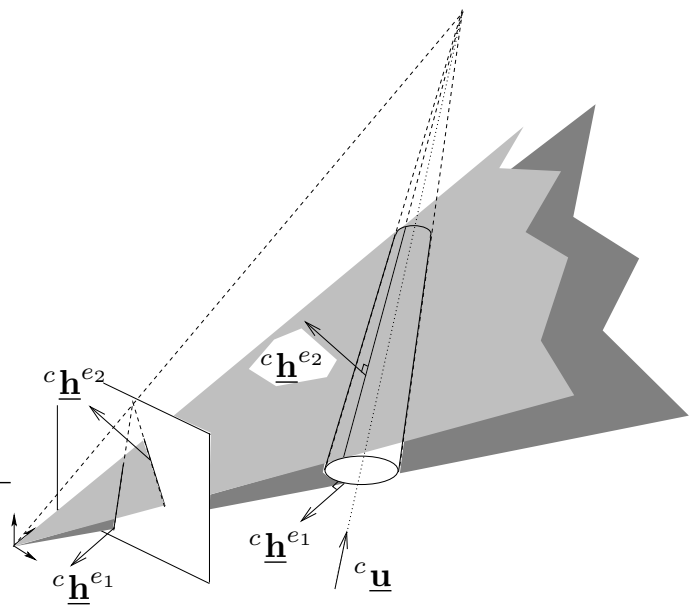

Figure 2: Projection of a cylinder in the image.

a joint sensor delivers a value expressed in its own reference frame. To convert this value in the base frame would require either an extremely accurate assembly procedure or the identification of the relative orientation of each joint sensor frame with respect to the base frame. Moreover, additional joint offsets would need be identified.

Since the leading vector of a leg is essentially a Cartesian feature, we chose to estimate it by vision. Indeed, vision is an adequate tool for Cartesian sensing, and, following (Renaud et al., 2004), if vision is also chosen for calibration, this does not add extra calibration parameter. It will even be shown in Section 4 that using vision reduces the parameter set needed for control.

\subsection{Cylindrical leg observation}

Now the problem is to recover ${ }^{c} \mathbf{u}_{i}$ from the leg observation. It may be somehow tedious, although certainly feasible, in the case of an arbitrary shape. Hopefully, for mechanical reasons such as rigidity, most of the parallel mechanisms are not only designed with slim and rectilinear legs, but, even better, with cylindrical shapes.

Except in the degenerated case where the projection center lies on the cylinder axis, the visual edge of a cylinder is a straight line (Figure 2). Consequently, it can be represented by its Binormalized Plücker coordinates (Andreff et al., 2002) in the camera frame. Let us note ${ }^{c} \underline{\mathbf{h}}^{e_{1}}$ and ${ }^{c} \underline{\mathbf{h}}^{e_{2}}$ the image projections of the two edges of a cylinder, that can be tracked using the results in (Marchand and Chaumette, 2005). These two vectors are oriented so that they point from the cylinder revolution axis outwards. Then, their expression is related to the Binormalized Plücker Coordinates $\left({ }^{c} \underline{\mathbf{u}},{ }^{c} \underline{\mathbf{h}},{ }^{c} h\right)$ of the cylinder axis (see Figure 3 ) 


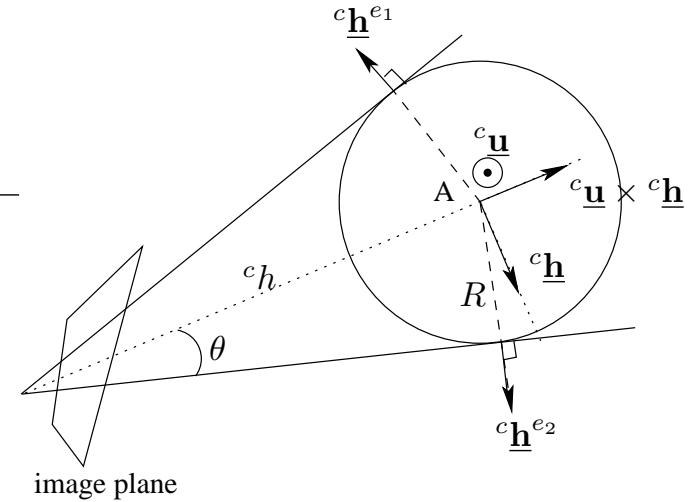

Figure 3: Construction of the visual edges of a cylinder: the cylinder is viewed from the top.

by

$$
\begin{aligned}
& { }^{c} \underline{\mathbf{h}}^{e_{1}}=-\cos \theta^{c} \underline{\mathbf{h}}-\sin \theta^{c} \underline{\mathbf{u}} \times{ }^{c} \underline{\mathbf{h}} \\
& { }^{c} \underline{\mathbf{h}}^{e_{2}}=+\cos \theta^{c} \underline{\mathbf{h}}-\sin \theta^{c} \underline{\mathbf{u}} \times{ }^{c} \underline{\mathbf{h}}
\end{aligned}
$$

where $\cos \theta=\sqrt{{ }^{c} h^{2}-R^{2}} /{ }^{c} h$ and $\sin \theta=R /{ }^{c} h$, ${ }^{c} h=\left\|{ }^{c} \mathbf{A} \times{ }^{c} \underline{\mathbf{u}}\right\|,{ }^{c} \underline{\mathbf{h}}=\left({ }^{c} \mathbf{A} \times{ }^{c} \underline{\mathbf{u}}\right) /{ }^{c} h$ and $R$ is the cylinder radius.

Then, it is easy to show that the leading vector ${ }^{c} \underline{\mathbf{u}}$ of the cylinder axis, expressed in the camera frame, writes

$$
{ }^{c} \underline{\mathbf{u}}=\frac{{ }^{c} \underline{\mathbf{h}}^{e_{1}} \times{ }^{c} \underline{\mathbf{h}}^{e_{2}}}{\left\|\underline{\mathbf{h}}^{e_{1}} \times{ }^{c} \underline{\mathbf{h}}^{e_{2}}\right\|}
$$

Notice that the geometric interpretation of this result is that ${ }^{c} \underline{\mathbf{u}}$ is, up to a scale factor, the vanishing point of the two image edges, i.e. their intersection point in the image.

\section{VISION-BASED CALIBRATION}

As stated earlier in this paper, the vision-based control (2)-(11) relies on the use of a calibrated camera and on some kinematic parameters.

The classical approach for calibrating a camera is to use a calibration grid made of points (Brown, 1971; Tsai, 1986; Faugeras and Toscani, 1987; Lavest et al., 1998). However, in the present case, it is more pertinent to use the method proposed in (Devernay and Faugeras, 2001). Indeed, this method is based on the observation of a set of lines, without any assumption on their 3D structure. As can be seen in Figure 1, there are plenty of lines in the images observed by a camera placed in front of a Gough-Stewart parallel robot. Hence, the camera can be calibrated on-line without any experimental burden.

Now, the only kinematic parameters the control law depends on are the attachment points of the legs onto the base expressed in the camera frame $\left({ }^{c} \mathbf{A}_{i}\right)$ and on the joint offsets. The latter appear in two places : under the form ${ }^{c} \mathbf{A}_{i}+q_{i}{ }^{c} \underline{\mathbf{u}}_{i}$ in (8) and as a gain in (9). Considering the order of magnitude of ${ }^{c} \mathbf{A}_{i}$ and $q_{i}$, one can neglect small errors on the joint offsets in (8). Moreover, since the joints are prismatic it is easy to measure their offsets manually with a millimetric accuracy. This is also highly sufficient to ensure that the gain in (9) is accurate enough. This means that, as far as control is concerned, one only needs to identify the attachment points onto the base, but there is no need for identifying the other usual kinematic parameters: attachment points onto the mobile platform and joint offsets.

In (Renaud et al., 2004), a calibration procedure was proposed, using legs observation, where, in a first step, the points $\mathbf{A}_{i}$ were estimated in the camera frame, then in a second step were expressed in the base frame, and finally the others kinematic parameters where deduced. Essentially, only the first step of this procedure is needed.

This step is reformulated here in a more elegant way, using the Binormalized Plücker Coordinates of the cylinder edges (12), and minimizing an imagebased criterion.

Assuming that the attachment point $\mathbf{A}_{i}$ is lying on the revolution axis of the leg and referring again to Figure 3, one obtains, for any leg $i$ and robot configuration $j$,

$$
\begin{aligned}
{ }^{c} \underline{\mathbf{h}}_{i, j}^{e_{1}{ }^{T c}} \mathbf{A}_{i} & =-R \\
{ }^{c} \underline{\mathbf{h}}_{i, j}^{e_{2}{ }^{T c}} \mathbf{A}_{i} & =-R
\end{aligned}
$$

Stacking such relations for $n_{c}$ robot configurations yields the following linear system

$$
\left(\begin{array}{c}
{ }^{c} \underline{\mathbf{h}}_{i, 1}^{e_{1} T} \\
{ }^{c} \underline{\mathbf{h}}_{i, 1}^{e_{2} T} \\
\vdots \\
{ }^{c} \underline{\mathbf{h}}_{i, n_{c}}^{e_{1}} T \\
{ }^{c} \underline{\mathbf{h}}_{i, n_{c}}^{e_{2}} T
\end{array}\right){ }^{c} \mathbf{A}_{i}=\left(\begin{array}{c}
-R \\
-R \\
\vdots \\
-R \\
-R
\end{array}\right)
$$

which has a unique least-square solution if there are at least two configurations with different leg orientations.

The calibration procedure is hence reduced to a strict minimum. To improve its numerical efficiency, one should only take care to use robot configurations with the larger angles between each leg orientation. However, since the ${ }^{c} \mathbf{A}_{i}$ only appear in the interaction matrix, they do not require a very accurate estimation.

\section{RESULTS}

A commercial DeltaLab hexapod was simulated, such that ${ }^{b} \mathbf{A}_{2 k}=R_{b}\left(\begin{array}{c}\cos \left(k \frac{\pi}{3}+\alpha\right) \\ \sin \left(k \frac{\pi}{3}+\alpha\right) \\ 0\end{array}\right),{ }^{b} \mathbf{A}_{2 k+1}=$ 


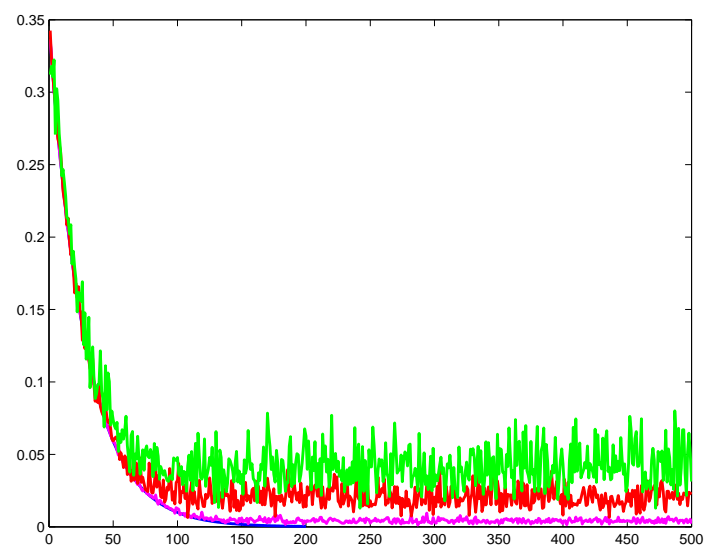

Figure 4: Robustness to noise : sum of squares of the errors $E^{T} E$ with a noise amplitude of $0 \mathrm{deg}, 0.01 \mathrm{deg}, 0.05 \mathrm{deg}$ and $0.1 \mathrm{deg}$.

$R_{b}\left(\begin{array}{c}\cos \left(k \frac{\pi}{3}-\alpha\right) \\ \sin \left(k \frac{\pi}{3}-\alpha\right) \\ 0\end{array}\right), \quad{ }^{e} \mathbf{B}_{2 k}=R_{e}\left(\begin{array}{c}\cos \left(k \frac{\pi}{3}+\beta\right) \\ \sin \left(k \frac{\pi}{3}+\beta\right) \\ 0\end{array}\right)$,

${ }^{e} \mathbf{B}_{2 k+1}=R_{e}\left(\begin{array}{c}\cos \left(k \frac{\pi}{3}-\beta\right) \\ \sin \left(k \frac{\pi}{3}-\beta\right) \\ 0\end{array}\right), k \in\{0,1,2\}$ with

$R_{b}=270 \mathrm{~mm}, \alpha=4.25^{\circ}, R_{e}=195 \mathrm{~mm}, \beta=$ $5.885^{\circ}$ and the legs range are $[345 \mathrm{~mm}, 485 \mathrm{~mm}]$.

\subsection{Image noise}

It is not immediate to model realistically the effect of image noise on detecting the visual edges of a cylinder. One can imagine to use the $(\rho, \theta)$ representation of an image line and to add noise to these two components. However, it is not certain that the two noise components are uncorrelated. Alternately, one can also determine the virtual intersections of the visual edge with the image border and to add noise to these intersections (Bartoli and Sturm, 2004).

An alternative model is presented here, which takes advantage of the fact that an image line is essentially a unit vector. Thus, image noise will necessary result into a rotation of this unit vector. Consequently, one needs to define a random rotation matrix, that is to say a rotation axis and a positive rotation angle. Preliminary tests showed that simply taking this axis as a uniformly distributed unit vector and this angle as a uniformly distributed positive scalar gives a realistic behavior.

This noise model has for advantages that it is easy to implement, it does not depend on the simulated image size, and is parametered by a single scalar (i.e. the maximal amplitude of the rotation angle).

To give an idea of how to choose this maximal amplitude, an error of about \pm 1 pixel on the extremities of a 300 pixel-long line segment yields a rotation angle of approximately 0.05 degree.

\subsection{Calibration validation}

In order to quantify the calibration procedure, the simulated robot was calibrated by moving it into its 64 extremal configurations (i.e. each leg joint is extended to its lower limit then to its upper limit). In each configuration, the visual edges of each leg were generated from the inverse kinematic model and added noise as described above, with a maximal amplitude of 0.05 degree. This calibration procedure was repeated 100 times. As a result, the median error on each attachment point is less than $1 \mathrm{~mm}$.

\subsection{Realistic simulation}

Now, a more realistic simulation is presented where the calibration is first performed using the 64 extremal configurations, then control is launched using the calibrated values. Noise is added as above during image detection, with amplitudes of $0.01,0.05$ and 0.1 degree.

It is noticeable that the calibration errors (in terms of maximal error on each of the component of each attachment point) is respectively of $0.5,1.4$ and 10 $\mathrm{mm}$.

Graphically (Figure 4), the sum of the errors on each leg $E^{T} E$ still decreases. Additionally, the median positioning error of the convergence tails in Figure 4 are respectively $0.1,0.6$ and $1.1 \mathrm{~mm}$ while the maximal error are 0.6, 1.9 and $3 \mathrm{~mm}$.

Hence, the overall calibration-control process seems rather robust to image measurement errors.

\section{CONCLUSION}

A fundamentally novel approach was proposed in (Andreff et al., 2005) for controlling a parallel mechanism using vision as a redundant sensor, adding a proprioceptive nature to the usual exteroceptive nature of vision. The present paper shows that such a method not only simplifies the control law, but also reduces the experimental effort.

Indeed, on the opposite to standard Cartesian control, no numerical inversion of the inverse kinematic model is required. Moreover, the kinematic parameter set which is effectively used during vision-based control is smaller than the one used for Cartesian control. Additionally, the kinematic calibration is turned linear with the vision-based approach.

From a practical point of view, setting-up such a control is made easier than any other ones. Indeed, on the opposite to most of the visual servoing approaches, there is no need to mount a visual target onto the robot end-effector, since the visual targets are the very robot legs. As far as calibration is concerned 
again, the calibration of the camera can be done with the same observation, as well as the kinematic calibration, which does not require any more any specific set-up.

Of course, all these benefits can only be obtained to a higher cost in software integration and development, which is not completely terminated at the present time, preventing us from showing experimental results.

\section{ACKNOWLEDGMENT}

This study was jointly funded by CPER Auvergne 2003-2005 program and by the CNRS-ROBEA program through the MP2 project.

\section{REFERENCES}

Andreff, N., Espiau, B., and Horaud, R. (2002). Visual servoing from lines. Int. Journal of Robotics Research, 21(8):679-700.

Andreff, N., Marchadier, A., and Martinet, P. (2005). Vision-based control of a Gough-Stewart parallel mechanism using legs observation. In Proc. Int. Conf. Robotics and Automation (ICRA'05), pages 25462551, Barcelona, Spain.

Baron, L. and Angeles, J. (1998). The on-line direct kinematics of parallel manipulators under joint-sensor redundancy. In Advances in Robot Kinematics, pages 126-137.

Bartoli, A. and Sturm, P. (2004). The 3d line motion matrix and alignement of line reconstructions. Int. Journal of Computer Vision, 57(3):159-178.

Brown, D. (1971). Close-range camera calibration. Photogrammetric Engineering, 8(37):855-866.

Christensen, H. and Corke, P., editors (2003). Int. Journal of Robotics Research - Special Issue on Visual Servoing, volume 22

DeMenthon, D. and Davis, L. (1992). Model-based object pose in 25 lines of code. Lecture Notes in Computer Science, pages 335-343.

Devernay, F. and Faugeras, O. (2001). Straight lines have to be straight. Machine Vision and Applications, 13:1424.

Dietmaier, P. (1998). The Stewart-Gough platform of general geometry can have 40 real postures. In Lenarčič, J. and Husty, M. L., editors, Advances in Robot Kinematics: Analysis and Control, pages 1-10. Kluwer.

Espiau, B., Chaumette, F., and Rives, P. (1992). A New Approach To Visual Servoing in Robotics. IEEE Trans. on Robotics and Automation, 8(3).

Faugeras, O. and Toscani, G. (Tokyo, japan, 1987). Camera calibration for $3 \mathrm{~d}$ computer vision. In International Conference on Computer Vision and Pattern Recognition $(C V P R)$.
Gogu, G. (2004). Fully-isotropic T3R1-type parallel manipulator. In Lenarčič, J. and Galletti, C., editors, On Advances in Robot Kinematics, volume Kluwer Academic Publishers, pages 265-272.

Husty, M. (1996). An algorithm for solving the direct kinematics of general Gough-Stewart platforms. Mech. Mach. Theory, 31(4):365-380.

Kallio, P., Zhou, Q., and Koivo, H. N. (2000). Threedimensional position control of a parallel micromanipulator using visual servoing. In Nelson, B. J. and Breguet, J.-M., editors, Microrobotics and Microassembly II, Proceedings of SPIE, volume 4194, pages 103-111.

Kim, H. and Tsai, L.-W. (2002). Evaluation of a Cartesian parallel manipulator. In Lenarčič, J. and Thomas, F., editors, Advances in Robot Kinematics: Theory and Applications. Kluwer Academic Publishers.

Koreichi, M., Babaci, S., Chaumette, F., Fried, G., and Pontnau, J. (1998). Visual servo control of a parallel manipulator for assembly tasks. In 6th Int. Symposium on Intelligent Robotic Systems, SIRS'98, pages 109-116, Edimburg, Scotland.

Lavest, J., Viala, M., and Dhome, M. (1998). Do we really need an accurate calibration pattern to achieve a reliable camera calibration. In Proceedings of ECCV98, pages 158-174, Freiburg, Germany.

Marchand, E. and Chaumette, F. (2005). Feature tracking for visual servoing purposes. Robotics and $\mathrm{Au}$ tonomous Systems.

Marquet, F. (2002). Contribution à l'étude de l'apport de la redondance en robotique parallèle. $\mathrm{PhD}$ thesis, LIRMM - Univ. Montpellier II.

Martinet, P., Gallice, J., and Khadraoui, D. (1996). Vision based control law using $3 \mathrm{~d}$ visual features. In Proc. World Automation Congress, WAC'96, Robotics and Manufacturing Systems, volume 3, pages 497-502, Montpellier, France.

Merlet, J.-P. (1990). An algorithm for the forward kinematics of general 6 d.o.f. parallel manipulators. Technical Report 1331, INRIA.

Merlet, J.-P. (2000). Parallel robots. Kluwer Academic Publishers.

Renaud, P., Andreff, N., Gogu, G., and Martinet, P. (2004). On vision-based kinematic calibration of a StewartGough platform. In 11th World Congress in Mechanism and Machine Science (IFToMM2003), pages 1906-1911, Tianjin, China.

Tsai, R. (Miami, UsA, 1986). An efficient and accurate calibration technique for $3 \mathrm{~d}$ machine vision. In International Conference on Computer Vision and Pattern Recognition (CVPR), pages pp 364-374.

Wang, J. and Masory, O. (1993). On the accuracy of a Stewart platform - Part I : The effect of manufacturing tolerances. In Proc. ICRA93, pages 114-120.

Zhao, X. and Peng, S. (2000). Direct displacement analysis of parallel manipulators. Journal of Robotics Systems, 17(6):341-345. 